\title{
Electric Charge Rotating Around a Black Hole
}

\author{
Jorge Castiñeiras, Luís C. B. Crispino, Rodrigo Murta \\ Departamento de Física, Universidade Federal do Pará, 66075-110, Belém, PA, Brazil \\ and George E. A. Matsas \\ Instituto de Física Teórica, Universidade Estadual Paulista, \\ Rua Pamplona 145, 01405-900, São Paulo, SP, Brazil
}

(Received on 14 October, 2005)

\begin{abstract}
We consider an electric charge rotating around a Schwarzschild black hole. We compute, using quantum field theory in curved spacetime at the tree level, the power emitted by the rotating charge minimally coupled to the Maxwell field. We also compute how much of the radiation emitted by the swirling charge is absorbed by the black hole.
\end{abstract}

We are commemorating the first centennial of Special Relativity. Despite how exotic Special and General Relativity may have appeared at first glance, they have become fundamental tools for now-a-days precision astrophysics. It is impossible to understand the center of active galaxies, pulsars and probably gamma ray bursts without evoking objects as black holes and neutron stars. As astrophysics measurements become more and more accurate, the influence of the spacetime curvature in particle wave modes will also need to be considered to explain the experimental data properly. This can be naturally accomplished in the context of quantum field theory in curved spacetimes. To illustrate this, we analyze the radiation emitted by an electric charge rotating around a Schwarzschild black hole (see Ref. [1] for the emission of scalar radiation from a rotating source around a Schwarzschild black hole). We assume natural units $\hbar=c=G=1$ and metric signature $(+---)$.

Let us consider the line element of the Schwarzschild spacetime in the form

$$
d s^{2}=f(r) d t^{2}-f(r)^{-1} d r^{2}-r^{2} d \theta^{2}-r^{2} \sin ^{2} \theta d \phi^{2},
$$

where $f(r)=1-2 M / r$ and the electromagnetic field described by the Lagrangian density in the modified Feynman gauge

$$
\mathcal{L}=\sqrt{-g}\left[-\frac{1}{4} F_{\mu v} F^{\mu v}-\frac{1}{2} G^{2}\right]
$$

with $g=r^{2} \sin \theta, G \equiv \nabla^{\mu} A_{\mu}+K^{\mu} A_{\mu}$ and $K^{\mu}=(0, d f / d r, 0,0)$. The corresponding Euler-Lagrange equations are, thus,

$$
\nabla_{v} F^{\mu v}+\nabla^{\mu} G-K^{\mu} G=0
$$

which can be written in terms of components as

$$
\begin{aligned}
& \frac{1}{f} \partial_{t}^{2} A_{t}-\frac{f}{r^{2}} \partial_{r}\left(r^{2} \partial_{r} A_{t}\right)+\frac{1}{r^{2}} \tilde{\nabla}^{2} A_{t}=0, \\
& \frac{1}{f} \partial_{t}^{2} A_{r}-\frac{1}{f} \partial_{r}\left[\frac{f^{2}}{r^{2}} \partial_{r}\left(r^{2} A_{r}\right)\right]+\frac{1}{r^{2}} \tilde{\nabla}^{2} A_{r} \\
& +\frac{1}{f} \partial_{r}\left(\frac{f}{r^{2}}\right) \tilde{\nabla}^{a} A_{a}=0, \\
& \frac{1}{f} \partial_{t}^{2} A_{a}-\partial_{r}\left(f \partial_{r} A_{a}\right)+\frac{1}{r^{2}}\left[\tilde{\nabla}^{b}\left(\tilde{\nabla}_{b} A_{a}-\tilde{\nabla}_{a} A_{b}\right)\right. \\
& \left.+\partial_{a} \tilde{\nabla}^{b} A_{b}\right]+r^{2} \partial_{r}\left(\frac{f}{r^{2}}\right) \partial_{a} A_{r}=0 .
\end{aligned}
$$

Here $a$ and $b$ denote angular variables on the unit 2-sphere $S^{2}$ with metric $\tilde{\eta}_{a b}$ and inverse metric $\tilde{\eta}^{a b}$ [with signature $(--)$ ], $\tilde{\nabla}_{a}$ is the associated covariant derivative on $S^{2}, \tilde{\nabla}^{a} \equiv \tilde{\eta}^{a b} \tilde{\nabla}_{b}$ and $\tilde{\nabla}^{2} \equiv \tilde{\eta}_{a b} \tilde{\nabla}^{a} \tilde{\nabla}^{b}$.

We write the complete set of positive-frequency solutions of Eq. (3) with respect to the Killing field $\partial_{t}$ as

$$
A_{\mu}^{(\varepsilon n \omega l m)}=\zeta_{\mu}^{\varepsilon n \omega l m}(r, \theta, \phi) e^{-i \omega t}, \omega>0 .
$$

The index $\varepsilon$ stands for the four different polarizations. The pure gauge modes, $\varepsilon=\mathrm{G}$, are the ones which satisfy the gauge condition $G=0$ and can be written as $A_{\mu}^{(\mathrm{G} n \omega l m)}=\nabla_{\mu} \Lambda$, where $\Lambda$ is a scalar field. The physical modes, $\varepsilon=\mathrm{I}$, II, satisfy the gauge condition and are not pure gauge. The nonphysical modes, $\varepsilon=\mathrm{NP}$, do not satisfy the gauge condition. The modes incoming from the past null infinity $\mathcal{g}^{-}$are denoted by $n=\leftarrow$ and the modes incoming from the past event horizon $\mathrm{H}^{-}$are denoted by $n=\rightarrow$. The $l$ and $m$ are the angular momentum quantum numbers.

The physical modes can be written as

$$
\begin{aligned}
& A_{\mu}^{(\mathrm{I} n \omega l m)}=\left(0, \frac{\varphi_{\omega l}^{\mathrm{I} n}(r)}{r} Y_{l m}, \frac{f}{l(l+1)} \frac{d}{d r}\left[r \varphi_{\omega l}^{\mathrm{I} n}(r)\right]\right. \\
& \left.\times \partial_{\theta} Y_{l m}, \frac{f}{l(l+1)} \frac{d}{d r}\left[r \varphi_{\omega l}^{\mathrm{I} n}(r)\right] \partial_{\phi} Y_{l m}\right) e^{-i \omega t}
\end{aligned}
$$

and

$$
A_{\mu}^{(\mathrm{II} n \omega l m)}=\left(0,0, r \varphi_{\omega l}^{\mathrm{II} n}(r) Y_{\theta}^{l m}, r \varphi_{\omega l}^{\mathrm{II} n}(r) Y_{\phi}^{l m}\right) e^{-i \omega t}
$$

with $l \geqslant 1$ (since the gauge condition $G=0$ is not satisfied for $l=0$ ). The radial part of the physical modes satisfies the differential equation

$$
\left(\omega^{2}-V_{S}\right)\left[r \varphi_{\omega l}^{\lambda n}(r)\right]+f \frac{d}{d r}\left(f \frac{d}{d r}\left[r \varphi_{\omega l}^{\lambda n}(r)\right]\right)=0,
$$

where $\lambda=\mathrm{I}$, II and $V_{S}=f l(l+1) / r^{2}$ is the Schwarzschild scattering potential. $Y_{l m}$ and $Y_{a}^{l m}$ are scalar and vector spherical harmonics [2], respectively.

The conjugate momenta associated with the field modes are

$$
\Pi^{(i) \mu v} \equiv-\left.\left[F^{\mu v}+g^{\mu v} G\right]\right|_{A_{\mu}=A_{\mu}^{(i)}},
$$


where $(i)$ represents $(\varepsilon, n, \omega, l, m)$. The modes are then normalized through the generalized Klein-Gordon inner product [3] such that

$$
\left(A^{(\varepsilon n \omega l m)}, A^{\left(\varepsilon^{\prime} n^{\prime} \omega^{\prime} l^{\prime} m^{\prime}\right)}\right)=M^{\varepsilon \varepsilon^{\prime}} \delta_{n n^{\prime}} \delta_{l l^{\prime}} \delta_{m m^{\prime}} \delta\left(\omega-\omega^{\prime}\right)
$$

where the matrix $M^{\varepsilon \varepsilon^{\prime}}$ is given by

$$
M^{\varepsilon \varepsilon^{\prime}}=\left(\begin{array}{cccc}
1 & 0 & 0 & 0 \\
0 & 1 & 0 & 0 \\
0 & 0 & 0 & -1 \\
0 & 0 & -1 & -1
\end{array}\right)
$$

with $\varepsilon=(\mathrm{I}, \mathrm{II}, \mathrm{G}, \mathrm{NP})$.

In order to quantize the electromagnetic field, we demand the equal time commutation relations

$$
\begin{gathered}
{\left[\hat{A}_{\mu}(\mathbf{x}, t), \hat{A}_{v}\left(\mathbf{x}^{\prime}, t\right)\right]=\left[\hat{\Pi}_{\mu t}(\mathbf{x}, t), \hat{\Pi}_{v t}\left(\mathbf{x}^{\prime}, t\right)\right]=0,} \\
{\left[\hat{A}_{\mu}(\mathbf{x}, t), \hat{\Pi}^{t \nu}\left(\mathbf{x}^{\prime}, t\right)\right]=\frac{i \delta_{\mu}^{v}}{\sqrt{-g}} \delta^{3}\left(\mathbf{x}-\mathbf{x}^{\prime}\right) .}
\end{gathered}
$$

The electromagnetic field operator can be expanded in terms of the normal modes as

$$
\hat{A}_{\mu}=\sum_{\varepsilon, n, l, m}^{\mathrm{Z}_{\infty}} d \omega\left[\hat{a}_{(i)} A_{\mu}^{(i)}+\hat{a}_{(i)}^{\dagger} \overline{A_{\mu}^{(i)}}\right]
$$

where $\hat{a}_{(i)}$ and $\hat{a}_{(i)}^{\dagger}$ are the annihilation and creation operators, respectively, satisfying

$$
\left[\hat{a}_{(\varepsilon n \omega l m)}, \hat{a}_{\left(\varepsilon^{\prime} n^{\prime} \omega^{\prime} l^{\prime} m^{\prime}\right)}^{\dagger}\right]=\left(M^{-1}\right)_{\varepsilon \varepsilon^{\prime}} \delta_{n n^{\prime}} \delta_{l l^{\prime}} \delta_{m m^{\prime}} \delta\left(\omega-\omega^{\prime}\right) .
$$

The Fock space of the physical states $|\mathrm{PS}\rangle$ is obtained by imposing the Gupta-Bleuler condition [4]. In our case, this corresponds to impose $\hat{a}_{(\mathrm{NP} \omega n l m)}|\mathrm{PS}\rangle=0$. The physical states are obtained by applying any number of creation operators $\hat{a}_{(\mathrm{I} n \omega l m)}^{\dagger}, \hat{a}_{(\mathrm{II} n \omega l m)}^{\dagger}$ and $\hat{a}_{(\mathrm{NP} n \omega l m)}^{\dagger}$ to the Boulware vacuum $|0\rangle$ defined by $\hat{a}_{(\varepsilon n \omega l m)}|0\rangle=0$. The creation operators associated with pure gauge modes take physical states into nonphysical ones. Moreover physical states of the form $\hat{a}_{(\mathrm{NP} n \omega l m)}^{\dagger}|\mathrm{PS}\rangle$ have zero norm. Therefore we can take as the representative elements of the Fock space those states obtained by applying the creation operators associated with the two physical modes to the Boulware vacuum. For this reason we will be concerned only with the two physical modes, $\lambda=\mathrm{I}$, II, in this paper. (A more detailed discussion of the Gupta-Bleuler quantization of the electromagnetic field in spherically symmetric and static spacetimes can be found in Ref. [5].)

The solutions of Eq. (10) are functions whose properties are not well known. (See Ref. [6] for some properties.) We can, however, obtain their analytic form (i) in the asymptotic regions for any frequency and (ii) everywhere if we keep restricted to the low-frequency regime. In order to study the asymptotic behavior of the physical modes we use the Wheeler coordinate $x=r+2 M \ln (r / 2 M-1)$ and rewrite Eq. (10) as

$$
\left(\omega^{2}-V_{S}\right)\left[r \varphi_{\omega l}^{\lambda n}(x)\right]+\frac{d^{2}}{d x^{2}}\left[r \varphi_{\omega l}^{\lambda n}(x)\right]=0 .
$$

Since the Schwarzschild potential vanishes for $r=2 M$ and decreases as $1 / r^{2}$ for $r \gg 2 M$, the solutions of Eq. (18) can be approximated in the asymptotic regions by

$$
r \varphi_{\omega l}^{\lambda \rightarrow}(r) \approx\left\{\begin{array}{cc}
B_{\omega l}^{\lambda \rightarrow}\left(e^{i \omega x}+R_{\omega l}^{\lambda \rightarrow} e^{-i \omega x}\right) & (x \ll-1), \\
B_{\omega l}^{\lambda \rightarrow} T_{\omega l}^{\lambda \rightarrow} i^{l+1} \omega x h_{l}^{(1)}(\omega x) & (x \gg 1),
\end{array}\right.
$$

and

$$
r \varphi_{\omega l}^{\lambda \leftarrow}(r) \approx\left\{\begin{array}{cc}
B_{\omega l}^{\lambda \leftarrow} T_{\omega l}^{\lambda \leftarrow} e^{-i \omega x} & (x \ll-1), \\
B_{\omega l}^{\lambda \leftarrow}\left((-i)^{l+1} \omega x h_{l}^{(1) *}(\omega x)\right. & \\
\left.+R_{\omega l}^{\lambda \leftarrow} i^{l+1} \omega x h_{l}^{(1)}(\omega x)\right) & (x \gg 1),
\end{array}\right.
$$

where $r \varphi_{\omega l}^{\lambda \rightarrow}(r)$ and $r \varphi_{\omega l}^{\lambda \leftarrow}(r)$ are solutions incoming from $H^{-}$ and $\mathcal{I}^{-}$, respectively. Here $h_{l}^{(1)}(x)$ is a spherical Bessel function of the third kind [7], $B_{\omega l}^{\lambda n}$ are normalization constants, and $\left|R_{\omega l}^{\lambda n}\right|^{2}$ and $\left|T_{\omega l}^{\lambda n}\right|^{2}$ are the reflexion and transmission coefficients, respectively, satisfying the usual probability conservation equation $\left|R_{\omega l}^{\lambda n}\right|^{2}+\left|T_{\omega l}^{\lambda n}\right|^{2}=1$. The normalization constants $B_{\omega l}^{\lambda n}$ are obtained using the generalized Klein-Gordon inner product defined above (see Ref. [8]).

Let us now find the analytic expressions of the physical modes in the low-frequency approximation. For this purpose we rewrite Eq. (10) as

$$
\begin{aligned}
& \frac{d}{d z}\left[\left(1-z^{2}\right) \frac{d \varphi_{\omega l}^{\lambda n}(z)}{d z}\right] \\
& +\left[l(l+1)-\frac{2}{z+1}-\omega^{2} M^{2} \frac{(z+1)^{3}}{z-1}\right] \varphi_{\omega l}^{\lambda n}(z)=0,
\end{aligned}
$$

where $z \equiv r / M-1$. In the low-frequency regime, we write the two independent solutions of Eq. (21) for $l \geqslant 1$ as

$$
\varphi_{\omega l}^{\lambda \rightarrow}(z) \approx C_{\omega l}^{\lambda \rightarrow}\left[Q_{l}(z)-\frac{(z-1)}{l(l+1)} \frac{d Q_{l}(z)}{d z}\right]
$$

and

$$
\varphi_{\omega l}^{\lambda \leftarrow}(z) \approx C_{\omega l}^{\lambda \leftarrow}\left[P_{l}(z)-\frac{(z-1)}{l(l+1)} \frac{d P_{l}(z)}{d z}\right],
$$

where $P_{l}(z)$ and $Q_{l}(z)$ are Legendre functions of the first and second kind [7], respectively, and $C_{\omega l}^{\lambda n}$ are normalization constants, which are obtained by fitting asymptotically Eqs. (22) and (23) with Eqs. (19) and (20), respectively [8].

Now let us consider an electric charge with $\theta=\pi / 2, r=$ $R_{S}$ and angular velocity $\Omega \equiv d \phi / d t=$ const $>0$ (as defined by asymptotic static observers), in uniform circular motion around a Schwarzschild black hole, described by the current density

$$
j_{S}^{\mu}\left(x^{\nu}\right)=\frac{q}{\sqrt{-g} u^{0}} \delta\left(r-R_{S}\right) \delta(\theta-\pi / 2) \delta(\varphi-\Omega t) u^{\mu} .
$$

Here $q$ is the coupling constant and

$$
u^{\mu}\left(\Omega, R_{S}\right)=\frac{1}{\sqrt{f\left(R_{S}\right)-R_{S}^{2} \Omega^{2}}}(1,0,0, \Omega)
$$




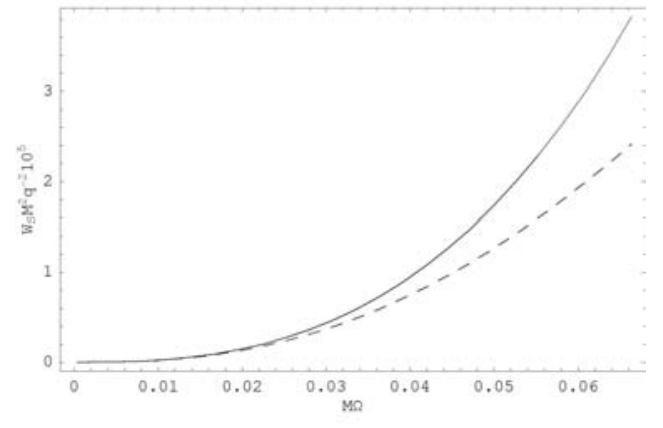

FIG. 1: The total power $W_{S}$ emitted by the electric charge rotating around a Schwarzschild black hole is plotted as a function of the angular velocity $\Omega$ as measured by asymptotic static observers. The solid line represents our numerical result whereas the dashed line represents our analytic result for low frequencies. The $l$ summation in Eq. (28) is performed up to $l=6 . M \Omega$ ranges from 0 up to 0.068 (associated with the innermost stable circular orbit at $R_{S}=6 M$ ).

is the charge's 4-velocity. We note that $j_{S}^{\mu}$ is conserved, $\nabla_{\mu} j_{S}^{\mu}=0$, and thus ${ }_{\Sigma}^{\mathrm{R}} d \Sigma_{\mu}^{(3)} j_{S}^{\mu}\left(x^{\nu}\right)=q$ for any Cauchy surface $\Sigma$.

Next let us minimally couple the charge to the field through the Lagrangian $\mathcal{L}_{I}=\sqrt{-g} j_{S}^{\mu} \hat{A}_{\mu}$. Then the emission amplitude at the tree level of one photon with polarization $\varepsilon$ and quantum numbers $(n, \omega, l, m)$ into the Boulware vacuum is given by

$$
\mathcal{A}^{\varepsilon n \omega l m}=i^{\mathrm{Z}} \quad d^{4} x \sqrt{-g} j_{S}^{\mu} \overline{A_{\mu}^{(\varepsilon n \omega l m)}} .
$$

It can be shown that $\mathcal{A}^{\varepsilon n \omega l m} \propto \delta(\omega-m \Omega)$. This implies that only photons with frequency $\omega_{0}=m \Omega$ are emitted once the charge has some fixed $\Omega=$ const. One can also verify that the pure gauge and nonphysical modes have vanishing emission amplitudes. This is so for the pure gauge modes because $\nabla_{\mu} j_{S}^{\mu}=0$ and for the nonphysical modes because they have zero norm.

The total emitted power is

$$
W_{S}=\sum_{\lambda=\mathrm{I}, \mathrm{II}} \sum_{n=\leftarrow, \rightarrow l=1} \sum_{m=1}^{\infty} \sum_{m}^{l}{ }^{\mathrm{Z}} d \omega \omega\left|\mathcal{A}^{\lambda n \omega l m}\right|^{2} / T,
$$

where $T=2 \pi \delta(0)$ is the total time as measured by the asymptotic static observers. Using now Eqs. (8)-(9) and (24)-(25) we rewrite Eq. (27) as

$$
W_{S}=\sum_{n=\leftarrow, \rightarrow l=1} \sum_{m=1}^{\infty} \sum_{m}^{l}\left[W_{S}^{\mathrm{I} n \omega_{0} l m}+W_{S}^{\mathrm{II} n \omega_{0} l m}\right]
$$

with

$$
\begin{aligned}
& W_{S}^{\mathrm{I} n \omega_{0} l m}=\frac{2 \pi q^{2} m^{3} \Omega^{3}}{[l(l+1)]^{2}}\left(1-\frac{2 M}{R_{S}}\right)^{2} \\
& \times\left[\frac{d}{d R_{S}}\left[R_{S} \varphi_{\omega_{0} l}^{\mathrm{In}}\left(R_{S}\right)\right]\right]^{2}\left|Y_{l m}(\pi / 2,0)\right|^{2}
\end{aligned}
$$

and

$$
W_{S}^{\mathrm{II} n \omega_{0} l m}=2 \pi q^{2} m \Omega^{3}\left[R_{S} \varphi_{\omega_{0} l}^{\mathrm{II} n}\left(R_{S}\right)\right]^{2}\left|Y_{\phi}^{l m}(\pi / 2,0)\right|^{2} .
$$

According to General Relativity for a stable circular orbit around a Schwarzschild black hole we have $R_{S}=\left(M / \Omega^{2}\right)^{1 / 3}$. We use this relation to compute numerically the emitted power given by Eqs. (28)-(30) as a function of $\Omega$. The numerical method used here is analogous to the one described in Ref. [1]. The result is plotted as the solid line in Fig. 1. The main contribution to the total emitted power comes from modes with angular momentum $l=m=1$. As a general rule, (i) the smaller is the $l$, the larger is the contribution to the total radiated power, and (ii) for a fixed value of $l$, the larger is the $m$, the larger is the contribution to the total radiated power.

It is interesting to note that the magnitude of the total radiated power in the electromagnetic case is approximately twice the numerical result found previously for a scalar source coupled to a massless Klein-Gordon field [1]. In principle, this is not surprising because of the fact that photons have two physical polarizations. Notwithstanding, it should be emphasized that the two polarizations contribute quite differently to the emitted power. For our rotating charge, the contribution from mode $\lambda=$ II is negligible when compared with the one from mode $\lambda=\mathrm{I}$. In order to get a feeling about it, we first recall that although the physical modes I have a non-vanishing radial component in contrast to the physical modes II, our current is such that $j^{r}=0$. Thus, the radial component $A_{r}^{(\varepsilon n \omega l m)}$ of the physical modes do not contribute to the corresponding interaction amplitude. In addition, since both physical modes are such that $A_{t}^{(\varepsilon n \omega l m)}=0$, all their contributions to the emitted power come from the angular components of $A_{\mu}^{(\varepsilon n \omega l m)}$. Now, because $Y_{l m}(\pi / 2,0)$ and $Y_{\phi}^{l m}(\pi / 2,0)$ are non vanishing only for even and odd $l+m$, respectively, the dominant contribution to the emitted power should come from modes I, since only they contribute when $l=1$ (i.e. $W_{S}^{\mathrm{I} n \omega_{0} 11} \neq 0$ while $W_{S}^{\mathrm{IIn} n \omega_{0} 11}=0$ ), and for a fixed $l$ only they contribute when $m$ has the maximum allowed value (i.e. $W_{S}^{\mathrm{In} \omega_{0} l l} \neq 0$ while $W_{S}^{\mathrm{IIn} \omega_{0} l l}=0$ ) (see discussion in the previous paragraph), which complies with our quantitative results. Moreover, it is very interesting to note that because the physical modes I and II only contribute to $W_{S}^{\mathrm{In} \omega_{0} l m}$ and $W_{S}^{\mathrm{IIn} n \omega_{0} l m}$ when $l+m$ is even and $o d d$, respectively, this means that only physical modes, which lead asymptotically to electric fields oscillating at the orbit plane $\theta=\pi / 2$ along $(\partial / \partial \phi)^{\mu}$ contribute to the emitted power, which is a familiar fact from synchrotron radiation physics (see, e.g., Ref. [9]).

Next, we use our low-frequency expressions for the physical modes to obtain an analytic approximation for the emitted power. The result is plotted as the dashed line in Fig. 1. We see from it that the numerical and analytical results differ sensibly as the charge approaches the black hole but coincide asymptotically, since far away from the hole only low frequency modes contribute to the emitted power.

Now let us compare the power emitted by the swirling charge in Schwarzschild spacetime $\left(W_{S}\right)$, with the correspondent power emitted in Minkowski spacetime $\left(W_{M}\right)$, the latter being obtained assuming that the charge is moving in a circular orbit due to a Newtonian gravitational force around a central object with the same mass $M$ as the black hole. 


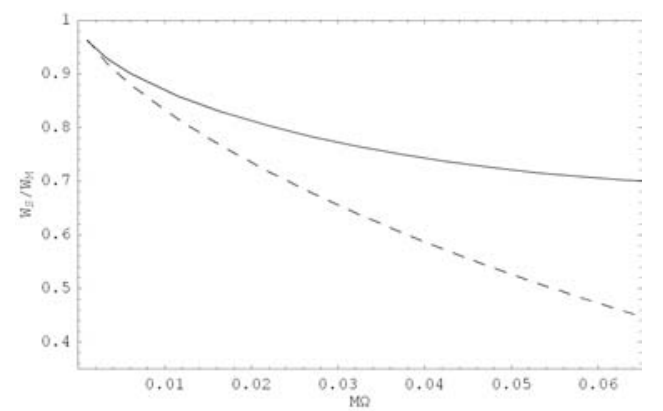

FIG. 2: The ratio $W_{S} / W_{M}$ is plotted as a function of the angular velocity $\Omega$. Again we consider contributions of the angular momentum up to $l=6$ in the summations. The maximum value of $M \Omega$ is 0.068 . The solid line corresponds to our numerical result, while the dashed line corresponds to our analytical low-frequency approximation result.

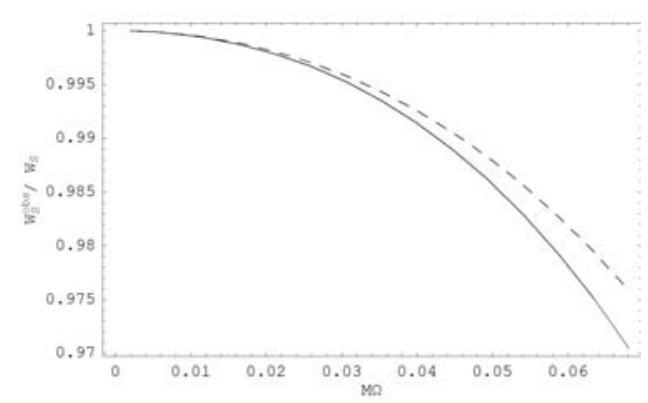

FIG. 3: The ratio between the asymptotically observed power $W_{S}^{\text {obs }}$ and the emitted power $W_{S}$ is plotted as a function of the angular velocity $\Omega$ of the swirling electric charge according to asymptotic static observers. $M \Omega$ varies from 0 to 0.068 . The summations in Eqs. (31) and (28) are performed up to $l=6$.

The quantization of the electromagnetic field in flat spacetime can be performed analogously to the procedure exhibited previously by making $f=1$. We then compare our curved and flat spacetime results as functions of the physical observables $M$ and $\Omega$ as measured by asymptotic static observers. We plot the ratio between $W_{S}$ and $W_{M}$ in Fig. 2 obtained from our numerical computations (solid line) and from our low-frequency analytic approximation (dashed line). In both cases the ratio tends to the unity as the charge rotates far away from the attractive center, as a consequence of the fact that the Schwarzschild spacetime is asymptotically flat. As the rotating charge approaches the central object, curved and flat spacetime results differ more significantly. In the innermost relativistic stable circular orbit, the numerical computation gives that $W_{S}$ is $30 \%$ smaller than $W_{M}$.

Now, this is interesting to use our quantum field theory in Schwarzschild spacetime approach to compute what is the amount of emitted radiation which can be asymptotically observed. This is given by

$$
\begin{array}{r}
W_{S}^{o b s}=\sum_{\lambda=\mathrm{I}, \mathrm{II}} \sum_{l=1}^{\infty} \sum_{m=1}^{l}\left[\left|T_{\omega_{0} l}^{\lambda \rightarrow}\right|^{2} W_{S}^{\lambda \rightarrow \omega_{0} l m}\right. \\
\left.+\left|R_{\omega_{0} l}^{\lambda \leftarrow}\right|^{2} W_{S}^{\lambda \leftarrow \omega_{0} l m}\right] .
\end{array}
$$

Our results are shown in Fig. 3. We see that the black hole absorbs only a small amount of the emitted radiation. Even for the innermost stable circular orbit the black hole absorbs only $3 \%$ of the total radiated power.

This is interesting and non trivial to make educated guesses about what are the most promising situations where the influence of the spacetime curvature on particle wave modes will be "essential" to understand the forthcoming observational data. We believe that black holes provide an excellent background stage and are, perhaps, the most natural candidates. Quantum field theory in Schwarzschild spacetime may become to tomorrow astrophysics as important as quantum field theory in Minkowski spacetime is to today particle physics.

The authors are grateful to Conselho Nacional de Desenvolvimento Científico e Tecnológico (CNPq) for partial financial support. R. M. and G. M. would like to acknowledge also partial financial support from Coordenação de Aperfeiçoamento de Pessoal de Nível Superior (CAPES) and Fundação de Amparo à Pesquisa do Estado de São Paulo (FAPESP), respectively.
[1] L. C. B. Crispino, A. Higuchi, and G. E. A. Matsas, Class. Quant. Grav. 17, 19 (2000).

[2] A. Higuchi, Class. Quant. Grav. 4, 721 (1987).

[3] L. C. B. Crispino, A. Higuchi, and G. E. A. Matsas, Phys. Rev. D 58, 084027 (1998).

[4] C. Itzykson and J. -B. Zuber, Quantum Field Theory (McGrawHill, New York, 1980).

[5] L. C. B. Crispino, A. Higuchi, and G. E. A. Matsas, Phys. Rev. D 63, 124008 (2001).
[6] B. P. Jensen and P. Candelas, Phys. Rev. D 33, 1590 (1986); 35, 4041(E) (1987).

[7] I. S. Gradshteyn and I. M. Ryzhik, Tables of Integrals, Series, and Products (Academic Press, New York, 1980).

[8] J. Castiñeiras, L. C. B. Crispino, G. E. A. Matsas, and R. Murta, Phys. Rev. D 71, 104013 (2005).

[9] A. A. Sokolov and I. M. Ternov, Radiation from Relativistic Electrons (American Institute of Physics, New York, Berlin, 1986). 